\title{
Protection of the external branch of the superior laryngeal nerve in Transoral endoscopic thyroidectomy vestibular approach (TOETVA): an observational study
}

\section{Zhen Wu}

Beijing Tongren Hospital, Capital Medical University

Jugao Fang ( $\square$ fangjugao2@ccmu.edu.cn )

Beijing Tongren Hospital, Capital Medical University

Hongzhi Ma

Beijing Tongren Hospital, Capital Medical University

Xiao Chen

Beijing Tongren Hospital, Capital Medical University

Qi Zhong

Beijing Tongren Hospital, Capital Medical University

Lizhen Hou

Beijing Tongren Hospital, Capital Medical University

Junwei Huang

Beijing Tongren Hospital, Capital Medical University

Ru Wang

Beijing Tongren Hospital, Capital Medical University

Xixi Shen

Beijing Tongren Hospital, Capital Medical University

\section{Research Article}

Keywords: External Branch of the Superior Laryngeal Nerve, Transoral Endoscopic, Papillary Thyroid Cancer, Protection Function, Sternothyroid Muscle

Posted Date: March 29th, 2021

DOl: https://doi.org/10.21203/rs.3.rs-275308/v1

License: (9) This work is licensed under a Creative Commons Attribution 4.0 International License. Read Full License 



\title{
Protection of the external branch of the superior laryngeal nerve in Transoral
}

\section{endoscopic thyroidectomy vestibular approach (TOETVA): an observational}

\section{study}

Zhen $W^{1,2}$, Jugao $\mathrm{F}^{1 *}$, Hongzhi $\mathrm{M}^{1}$, Xiao $\mathrm{C}^{1}$, Qi $Z^{1}$, Lizhen $\mathrm{H}^{1}$, Junwei $\mathrm{H}^{1}$, Ru $\mathrm{W}^{1}$, Xixi $\mathrm{S}^{1}$

1.Thyroid Center, Beijing Tongren Hospital, Capital Medical University, Beijing 100730, P.R. China;

2. Department of Thyroid and Breast Surgery, Liaocheng People's Hospital, Liaocheng hospital affiliated to Shandong first medical university, Liaocheng 252000, Shandong, China Correspondence to: Dr Jugao Fang, Thyroid Center, Beijing Tongren Hospital, Capital Medical University, Beijing 100730, P.R. China.

E-mail: fangjugao2@ccmu.edu.cn

\begin{abstract}
Background: Avoiding injury of the external branch of the superior laryngeal nerve (EBSLN) is one of the major challenges during thyroid surgery, especially in transoral endoscopic thyroidectomy vestibular approach (TOETVA). This study aimed to investigate the protective strategies of the EBSLN during TOETVA.

Methods: In order to protect the EBSLN during TOETVA, we adopted the method of identification the nerve by anatomy and localization. The method of anatomy involves the dissection of EBSLN by complete transection of the sternothyroid muscle in the attachment of the thyroid cartilage. The method of localization involves nerve stimulation localization, which produces cricothyroid contractile activity through intraoperative nerve monitoring stimulation (IONM). Concurrently, patients were evaluated preoperatively and at 1 and 3 weeks postoperatively in an individual prospective cohort study using a stroboscopic laryngoscope and the voice handicap index-10 (VHI-10). The VHI-10 score was used to evaluate voice changes.
\end{abstract}

Results: We retrospectively analyzed patients with papillary thyroid cancer (PTC) who underwent TOETVA in the thyroid center of the Beijing Tongren hospital between February 2018 and June 2020. Patients with recurrent laryngeal nerve (RLN) damage were excluded. Sixty patients were enrolled in this study, of which four underwent total thyroidectomy. Intraoperatively, 56 EBSLNs were located (56/64, 87.50\%). Among these, the left EBSLN was identified in 20/25(80.00\%) and the right EBSLN was identified in 36/39 (92.31\%) cases. One week postoperatively, a blinded stroboscopic laryngoscope examination showed that no patient had paresis of the EBSLN. However, the VHI-10 score was significantly higher than the preoperative value $(10.58 \pm 4.54$ vs. $3.00 \pm 1.54, p<0.01)$. At three weeks postoperatively, the overall score was still different from that preoperatively ( $4.83 \pm 3.34$ vs. $3.00 \pm 1.54, p<$ $0.01)$; however, the vast majority of patients returned to their preoperative status.

Conclusion: In TOETVA, the EBSLN can be well exposed by transection of the sternothyroid muscle, and combined with IONM, the protection of the function of the EBSLN can be guaranteed. Simultaneously, we observed that TOETVA could cause a short-term voice handicap in patients, with such changes generally returning to normal within three weeks. 
Keyword: External Branch of the Superior Laryngeal Nerve; Transoral Endoscopic; Papillary Thyroid Cancer; Protection Function; Sternothyroid Muscle

\section{Introduction}

Due to the advantage of leaving no scars, transoral endoscopic thyroidectomy (TOET) gradually developed in China ${ }^{[1]}$ and abroad and quickly became a hot research topic. In 2016, Anuwong ${ }^{[2]}$ reported for the first time the transoral endoscopic thyroidectomy vestibular approach (TOETVA). More and more reports indicate that TOETVA is a safe and cosmetic alternative for well-selected patients ${ }^{[2-5]}$. However, TOETVA still has some problems, including oncologic concerns of cancer seeding and local recurrence, the significant learning curve, and the potential for injury to the recurrent laryngeal nerve (RLN) or the mental nerve $(\mathrm{MN})^{[6-8]}$. Meanwhile, due to the problem of the TOETVA approach, the superior extreme of the thyroid is difficult to be exposed, and the injury of the external branch of the superior laryngeal nerve (EBSLN) may occur, but there are few reports in this regard ${ }^{[9-10]}$. Some scholars have pointed out that during TOETVA, the superior thyroid artery and vein are ligated as close to the thyroid as possible, and the EBSLN is preserved by avoiding its anatomical exposure ${ }^{[1]}$.

However, the EBSLN is in closed anatomical proximity to the superior thyroid vessels and there is heterogeneity in the anatomy of the EBSLN. The EBSLN can be easily injured when the vessels of the superior thyroid are separated close to the gland, especially when it is difficult to expose the superior pole of the thyroid from the perspective of TOETVA surgery in a cephalic to caudal manner ${ }^{[12]}$. In addition, in endoscopic surgery, only an ultrasonic device can be used to cut off the blood vessels. When used blindly, thermal damage by the ultrasonic device may cause damage to the EBSLN. We believe that dissection to expose the EBSLN may provide better protection. However, surgical dissection to locate the EBSLN may result in unnecessary trauma and adversely affect voice quality.

What are the more appropriate ways of protecting the EBSLN during TOETVA surgery? To address this question, this study was undertaken to identify the feasibility associated with a safe preservation of the EBSLN with transection of the sternothyroid muscle.

\section{Materials and methods}

\subsection{Patients}

This retrospective study was undertaken in the thyroid center of the Beijing Tongren hospital from February 2018 to June 2020. Inclusion criteria for patients followed the expert consensus of Chinese thyroid surgeons consisted of all patients diagnosed with papillary thyroid cancer by preoperative fine needle aspiration biopsy and those who underwent total thyroidectomy for bilateral micropapillary thyroid carcinoma. Exclusion criteria for this study included (1) patients with confirmed intraoperative injury of the RLN, and (2) patients who could not complete the follow-up task. This study was approved by our hospital. Written informed consent was obtained from each patient and the study was conducted in accordance with the Declaration of Helsinki.

\subsection{Surgical technique}

In this study, we completely transected the sternothyroid muscle near the attachment of the thyroid cartilage (Figure 1) and opened an avascular plane between the superior pole and 
the thyroid cartilage, which is called Joll's space [9] (Figure 2). In this space, the EBSLN could be identified beneath the thyroid suspensory ligament. Meanwhile, using intraoperative nerve monitoring stimulation (IONM) to stimulate the EBSLN at $1.0 \mathrm{~mA}$, we assessed the cricothyroid muscle (CTM) twitch and electromyography (EMG) response. IONM was realized by connecting the nerve monitor wire with the electrocoagulation hook. We could safely continue to dissect and ligate the superior thyroid vessels. If the CTM twitching was weak and the estimation of the location of the EBSLN was difficult, we would ligate the superior thyroid artery and vein as close to the superior thyroid pole as possible.

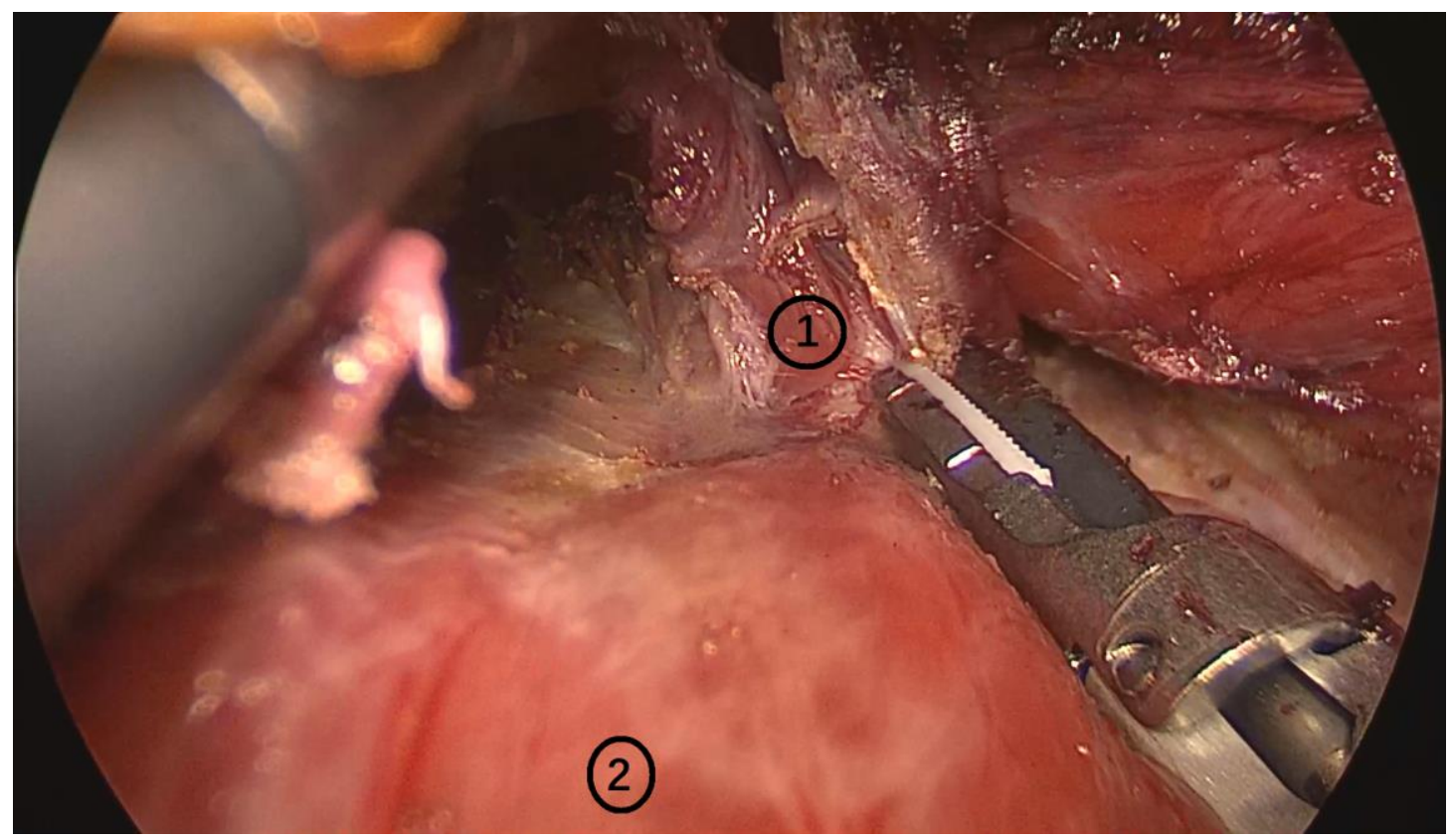

Figure 1 The sternothyroid muscle was transected at an attachment point on the thyroid cartilage, and was pushed to the sternal side, exposing the lobe of the thyroid gland. Here, (1) represents the transverse sternothyroid muscle, and (2) represents the thyroid gland lobe.

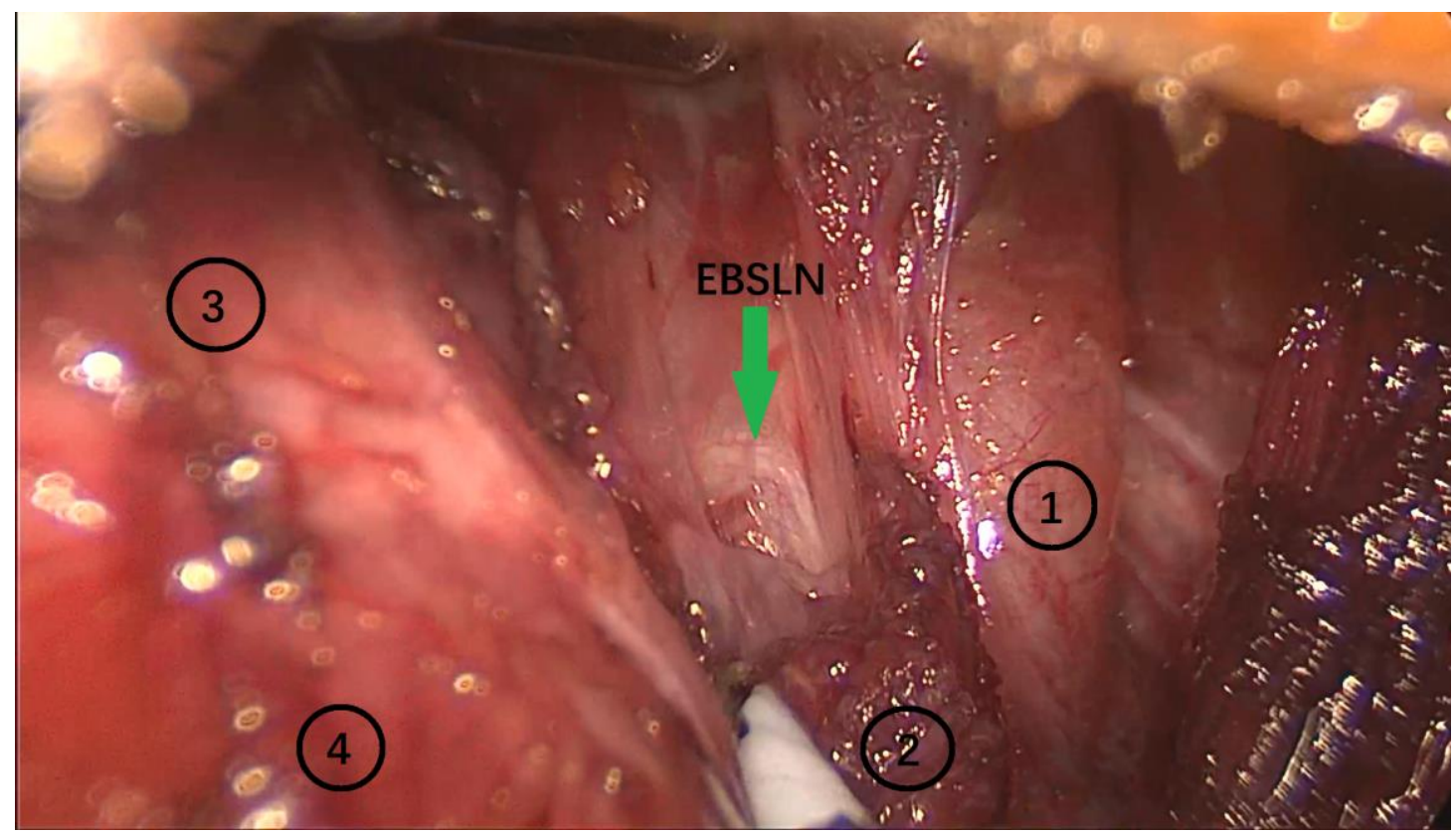

Figure 2 The thyroid gland was pulled outward and downward to expose Joll's space. In Joll's 
space, we looked for the EBSLN. (1) is the superior polar gland of the thyroid; (2) is the transverse sternothyroid muscle; (3) is the cricothyroid muscle (CTM) and (4) is the throat.

\subsection{Objective voice analysis}

Stroboscopic laryngoscopy was performed one week postoperatively, to further exclude any damage to the RLN and observe whether there was damage to the EBSLN. Patients with special symptoms were reevaluated three weeks later. We evaluated the voice changes in all patients using the voice handicap index-10 (VHI-10). Patients filled in the 10-item VHI-10 questionnaire $^{[13]}$. The $\mathrm{VHI}-10$ is one of the most psychometrically robust, well-studied, and efficient instruments among various instruments used to measure self-perceived voice related quality of life. The investigation time nodes were designed preoperatively, and at one and three weeks postoperatively.

\subsection{Statistics}

Statistical analysis was performed using SPSS 20.0 (IBM SPSS statistics 20.0.0; SPSS Inc, Chicago, IL). Continuous data are summarized as mean \pm interval of confidence $95 \%$. Vocal motility was evaluated with the Student's t-test, for paired sample test on parametric variables. $P$ values below 0.05 were considered significant.

\section{Results}

The data of 60 patients who underwent TOETVA were retrieved from the clinical database. All patients had papillary thyroid carcinoma and underwent central lymph node dissection. Four underwent total thyroidectomy and 56 underwent lobectomy. There were 50 females and 10 males, with an average age of $36.98 \pm 9.27$ years. Median operation time was 240.63 $\pm 66.39 \mathrm{~min}$. Mean postoperative drainage volume was $111.79 \pm 38.24 \mathrm{ml}$, and average postoperative hospital stay was $4.04 \pm 1.35$ days. The clinical data of all patients including postoperative complications are shown in Table 1.

In this study, left lobectomy was performed in 25 cases, and right lobectomy was performed in 39 cases. In all these 64 lobes, the EBSLN was identified in 56/64 cases (87.50\%) and unidentified in 8/64 (12.50\%) cases. Among these, the left EBSLN was identified in 20/25 (80.00\%) and the right EBSLN was identified in 36/39 (92.31\%) cases. CTM twitching was observed in all patients, and potentiometric wave images were present in some patients (Figure 3).

After completely transecting the sternothyroid muscle, the superior polar vessels of the thyroid could be severed under direct vision. Even the presence of superior pole vascular bleeding could be easily managed to avoid injury to the EBSLN (Figure 4).

One week postoperatively, stroboscopic laryngoscope monitoring showed that no patients had changes in vocal cord tension. In all patients, the VHI-10 score was significantly higher than that of the preoperative value $(10.58 \pm 4.54$ vs. $3.00 \pm 1.54, p<0.01)$. After three weeks, the overall score was still different from that preoperatively ( $4.83 \pm 3.34$ vs. $3.00 \pm 1.54, p<$ 0.01 ); however, the vast majority of patients returned to their preoperative status (Figure 5). 
Table 1 demographic data and operative details

\begin{tabular}{|c|c|}
\hline Characteristics and details & Value \\
\hline Age (median, years) & $36.98 \pm 9.27$ \\
\hline Sex (male/female) & $10 / 50$ \\
\hline Tumor location (left lobe/right lobe) & $21 / 39$ \\
\hline Operation time (median, min) & $240.63 \pm 66.39$ \\
\hline Postoperative drainage (median, $\mathrm{ml}$ ) & $111.79 \pm 38.24$ \\
\hline postoperative hospital stay (median, days) & $4.04 \pm 1.35$ \\
\hline \multicolumn{2}{|l|}{ Postoperative complication (cases) } \\
\hline infection & 1 \\
\hline seroma & 1 \\
\hline Skin damage & 1 \\
\hline Tracheal injury & 0 \\
\hline Postoperative bleeding & 0 \\
\hline Conjunctivitis & 1 \\
\hline Permanent RLN palsy & 0 \\
\hline Mental nerve injury & 0 \\
\hline Permanent hypoparathyroidism & 3 \\
\hline
\end{tabular}




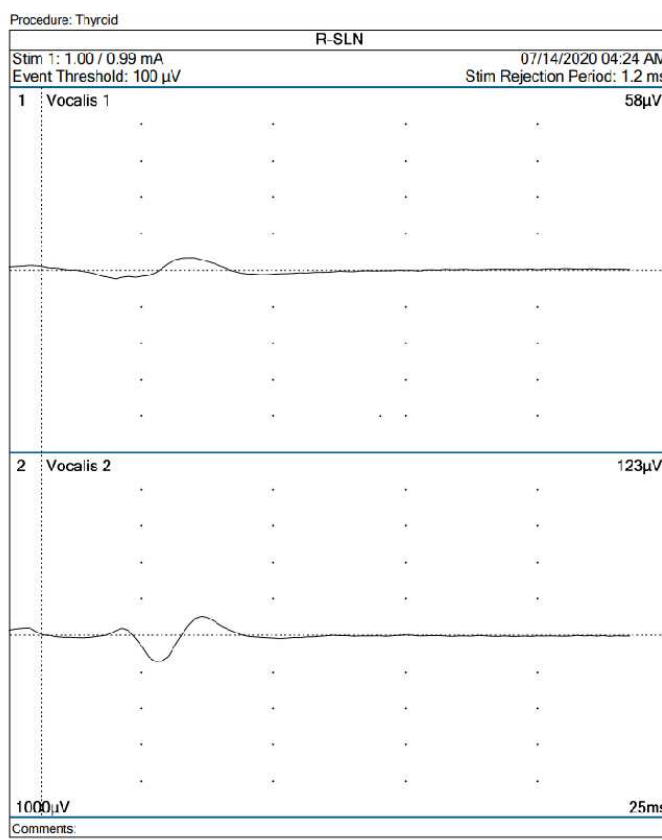

a

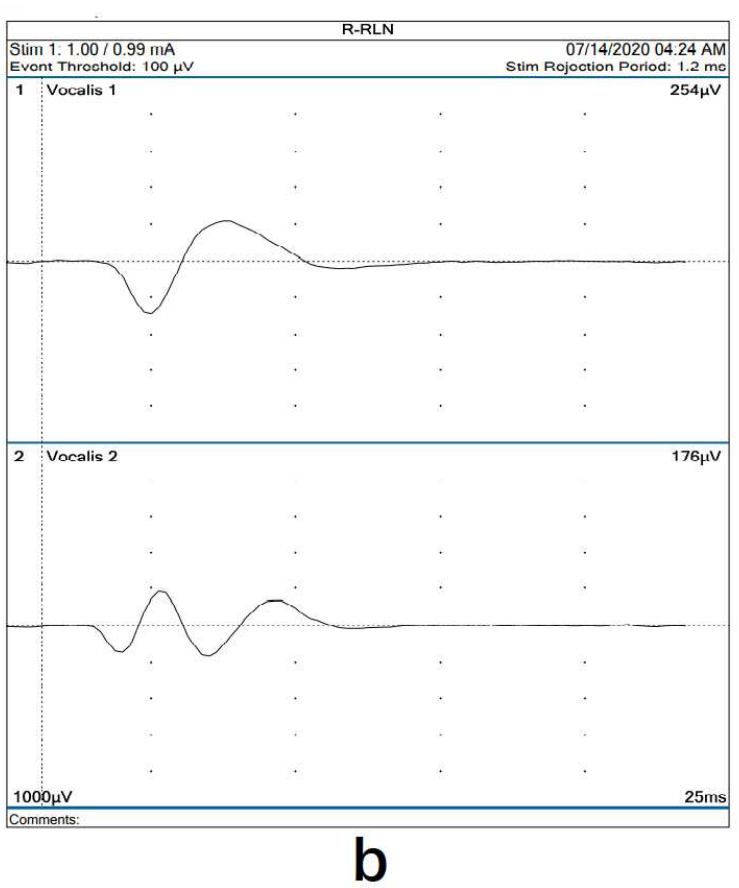

Figure 3 When IONM stimulated the EBSLN, CTM twitching was observed in all patients, and potentiometric wave images were present in some patients (see Figure $3 a$ ), and the waveform of the EBLSN was completely different from that of the RLN (see Figure 3b).

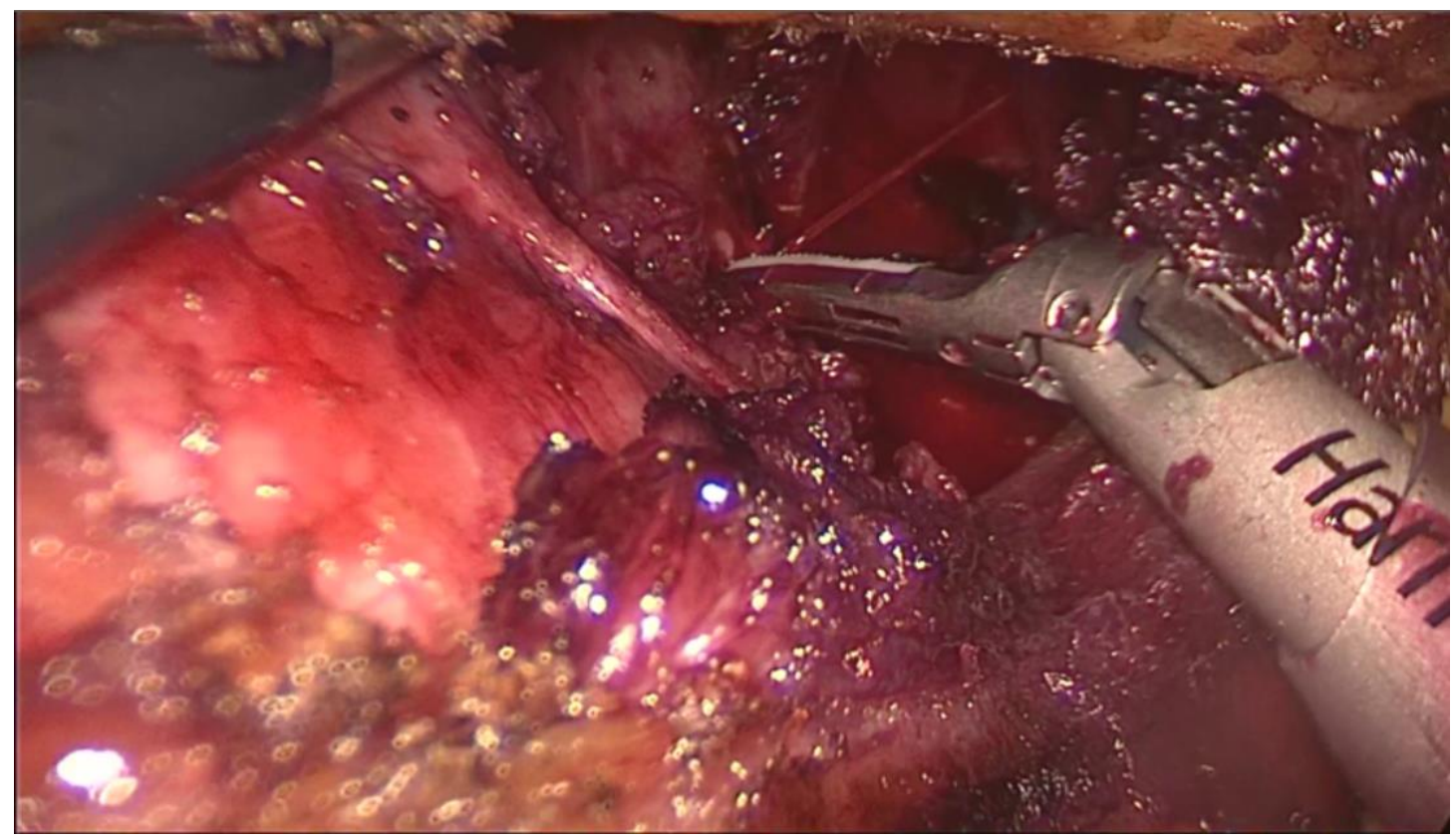

Fig $4 a$ 


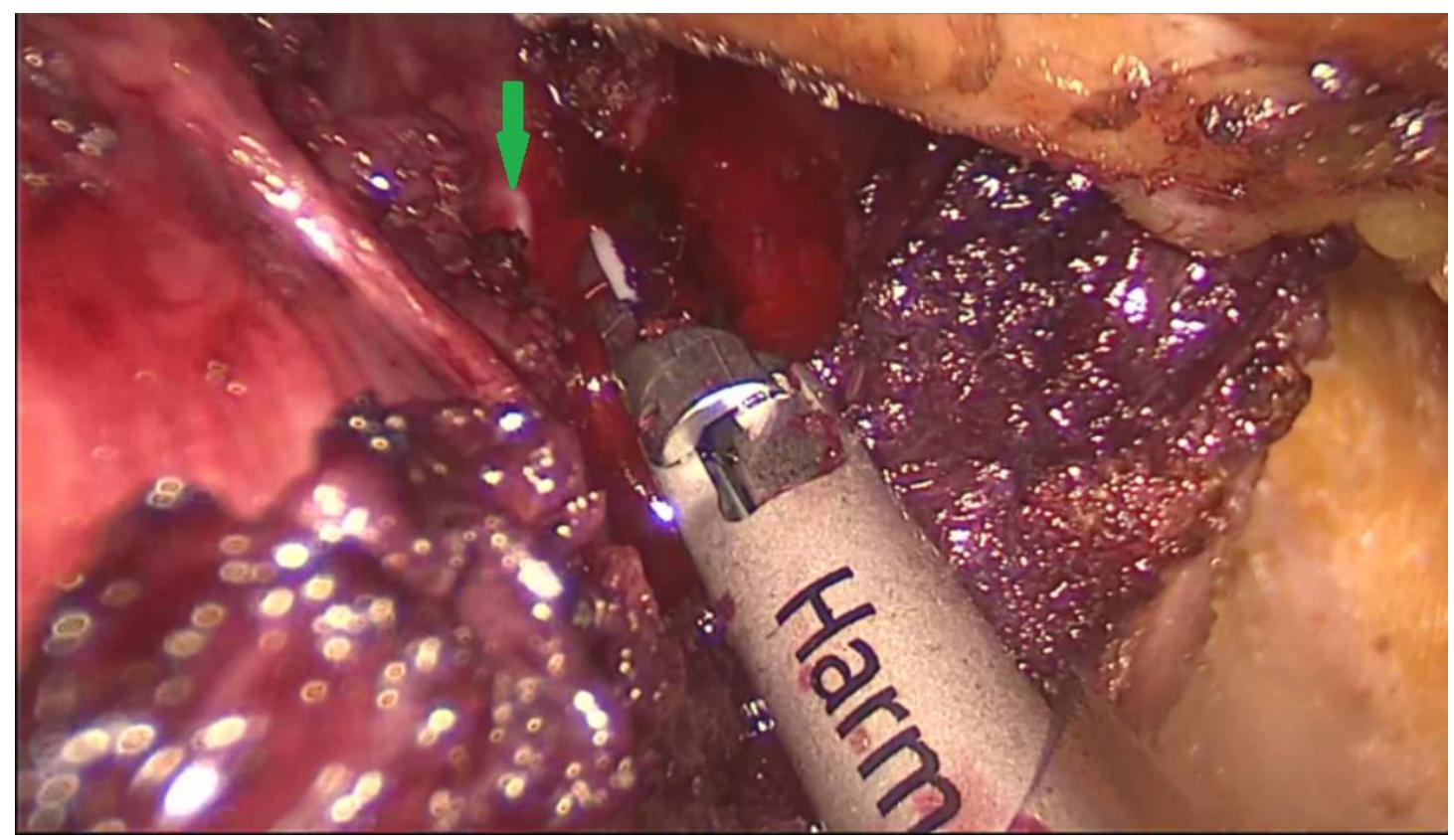

Fig4b

Figure 4 The bleeding point of the superior thyroid artery could be found under direct vision. The bleeding point could be clipped with an ultrasonic knife, and the non-functional head of the ultrasonic knife could be kept away from the EBSLN to avoid injury to the EBSLN. The arrow in the figure indicates the EBSLN.

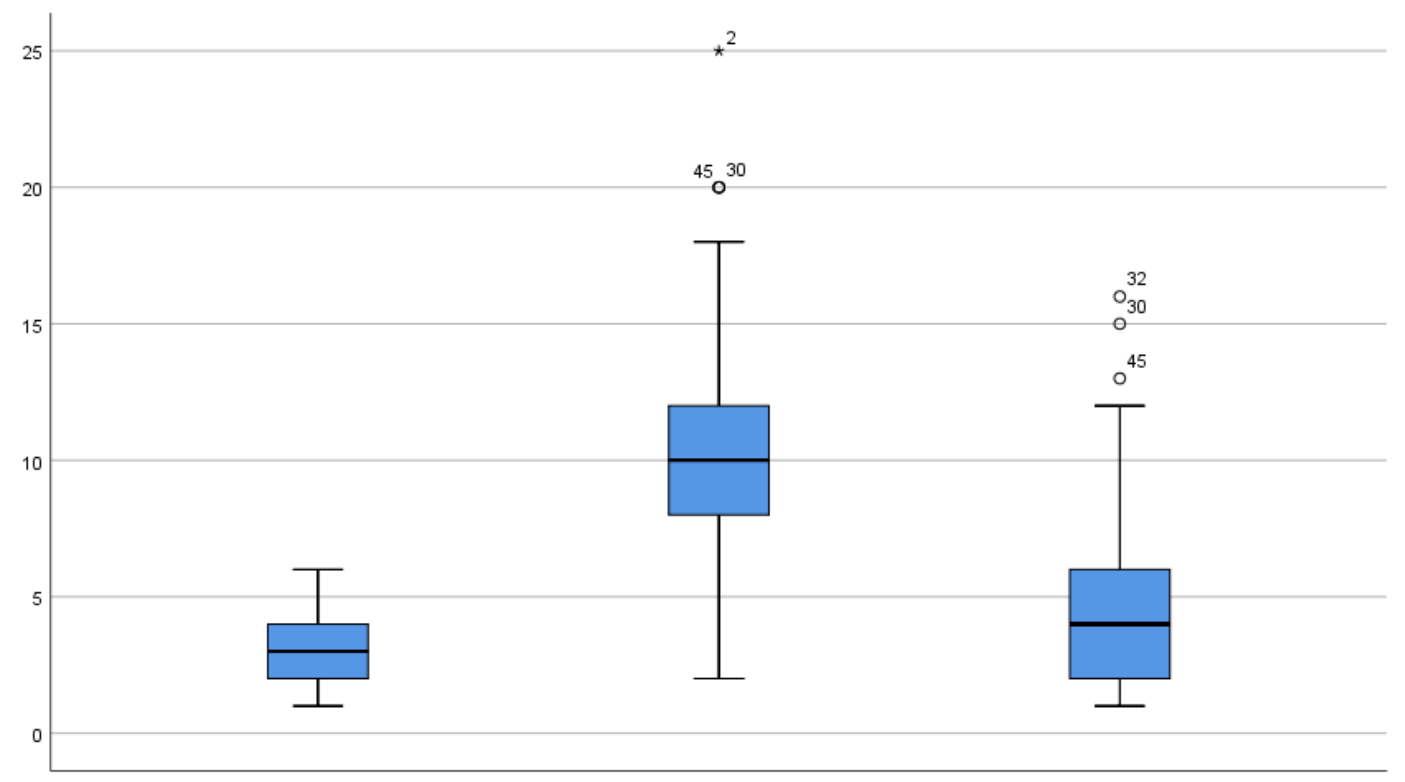

\section{Preoperative One week after surgery Three weeks after surgery}

Figure 5 Three weeks postoperatively, the vast majority of patients returned to their preoperative status.

\section{Discussion}

Due to the angle of approach, it is difficult to expose the upper pole of the thyroid during 
TOETVA surgery, which may lead to the injury of the EBSLN. Moreover, EBSLN has an average diameter of only $0.8 \mathrm{~mm}^{[14]}$ and is closely related to the vessels of the upper pole of the thyroid gland, which is prone to misbinding, thermal damage and even disconnection. The reported prevalence of EBSLN injury varies widely. It is reported in the literature that the EBSLN is injured in up to $58 \%$ of patients undergoing thyroidectomy ${ }^{[15]}$. Our previous study showed that in most cases (56\%), the EBSLN is located close to the thyroid superior pole under physiological conditions, and it is prone to be injured during thyroid surgery ${ }^{[16]}$.

However, EBSLN iatrogenic injury is considered the most commonly underestimated complication in thyroidectomy because vocal assessment underestimates such events and laryngoscopic postoperative evaluation does not show standardized findings ${ }^{[17]}$. Injury to the EBSLN can result in detrimental voice changes, which may impact the quality of voice with varying degrees of severity, which is more noticeable in professional voice users ${ }^{[18]}$. As the number of surgical patients, especially young women, increases every year, and postoperative quality of life becomes a major indicator of surgical success, more doctors realize that intraoperative protection of the EBSLN is as significant as that of the RLN. The protection of the EBSLN is very justified.

Recently, IONM provides multiple advantages in the EBSLN surgical approach. Several studies demonstrated that IONM was a more effective method than traditional visualization in localizing the EBSLN ${ }^{[19]}$. During EBSLN stimulation, CTM twitching is noted in patients. Therefore, some scholars have proposed that the process of dissecting exposed EBSLN may cause nerve damage, and the IONM region protection method can be used to protect the EBSLN. Close to the gland, the vessels in the upper pole of the thyroid are manipulated as if removing a hat ${ }^{[12]}$.

We believe that the treatment of the superior pole vessels closely attached to the gland may result in residual glands or inaccurate treatment of the superior pole vessels, resulting in postoperative bleeding. Furthermore, the use of a simple clingy gland treatment may also result in the disconnection of the EBSLN or in thermal damage by the ultrasonic scalpel. In particular, in some patients with superior pole vascular bleeding, such as the patients mentioned in this article, only the dissection of the EBSLN can avoid injury.

During surgery, we would cut off the sternothyroid muscle in the attachment of the thyroid cartilage, while pushing the muscle stump toward the head with the ultrasonic knife, concurrently, pulling the upper pole outwards and down with the uninjured grasping forceps. After sufficient separation of the body of the larynx from the superior polar gland, the EBSLN is usually exposed in an avascular area. The exposure rate of the EBSLN in our data was 87.50\%; and the right EBSLN was more exposed. We found that the anatomical exposure of the EBSLN could permit a safer manipulation of the superior pole vessels, even with hemorrhage of the superior pole vessels, it could be clipped safely and avoided injury to the EBSLN. At the same time, pushing the transverse sternothyroid muscle to the side of the sternum can better expose the thyroid gland and lymph nodes in the central region, which is conducive for lymph node dissection in the central region.

Previous studies have confirmed that the disconnection of the sternothyroid muscle has no effect on sound changes after thyroid surgery ${ }^{[20]}$. IONM provides regional protection for hard-to-view EBSLN. Cricothyroid muscle tremor was observed with $1 \mathrm{~mA}$ stimulation to indicate the location of the EBSLN. Our data shows that it is better to use both methods to 
protect the EBSLN. The steps to protect the EBSLN were outlined in the 2013 guidelines ${ }^{[14]}$ : (1) Expose of the space harboring the EBSLN; (2)Bluntly dissect the tissues; (3)Stimulate the tissues during dissection; (4)Look for a cricothyroid twitch; (5) Navigate your dissection using the technique of nerve mapping.

In addition, we found that after thyroid surgery, patients might present with pronunciation discomfort for a variety of reasons, possibly due to surgical stimulation or drainage tube placement. The $\mathrm{VHI}-10$ score was significantly higher 1 week postoperatively than the preoperative values. However, most patients returned to normal after 3 weeks. In our study, stroboscopic laryngoscope monitoring showed that no patients had changes in vocal cord tension.

\section{Conclusion}

In TOETVA, the EBSLN can be well exposed by transection of sternothyroid muscle, and combined with IONM, the function of the EBSLN can be well protected. At the same time, we observed that TOETVA could cause short-term voice handicap in patients, with such changes generally returning to normal within three weeks.

\section{Acknowledgement}

Not applicable.

\section{Authors' contributions}

ZW, HZM and XC participated in study design, operation technique and data analysis. JGF conceived the study. All authors read and approved the final manuscript.

\section{Funding}

My paper is supported by the Capital Health Research and Development of Special (Grant No. 2018-2-2054) ; meanwhile, this paper is supported by Wu Jieping Medical Foundation (Grant No. 320.6750.18229)

\section{Availability of data and materials}

The datasets used and/or analyzed during the current study available from the corresponding author on reasonable request.

\section{Ethics approval and consent to participate}

This study was approved by Capital Medical University affiliated Beijing Tongren hospital. Written informed consent was obtained from each patient and the study was conducted in accordance with the Declaration of Helsinki. Informed consents were received from the patient.

\section{Consent for publication}

Written informed consent was obtained from each patient.

\section{Competing interests}

The authors indicated no potential conflicts of interest.

\section{Reference}

[1]. Wang C, Zhai H, Liu W, et al. Thyroidectomy: A novel endoscopic oral vestibular approach[J]. Surgery, 2014, 155(1): 33-38.

[2]. Anuwong A. Transoral Endoscopic Thyroidectomy Vestibular Approach: A Series of the 
First 60 Human Cases[]]. World Journal of Surgery, 2016, 40(3): 491-497.

[3]. Liu Z, Peng X, Li Z, et al. Postoperative drain after transoral endoscopic thyroidectomy vestibular approach (TOETVA) with single incision[]]. Surgical Endoscopy and Other Interventional Techniques, 2020: 1-9.

[4]. Anuwong A, Ketwong K, Jitpratoom P, et al. Safety and Outcomes of the Transoral Endoscopic Thyroidectomy Vestibular Approach[]]. JAMA Surgery, 2017, 153(1): 21-27.

[5]. Russell J O, Anuwong A, Dionigi G, et al. Transoral Thyroid and Parathyroid Surgery Vestibular Approach: A Framework for Assessment and Safe Exploration[]]. Thyroid, 2018, 28(7): 825-829.

[6]. Yeh Y, Chen J, Kuo P, et al. Printing a Three-Dimensional Patient-Specific Safety Device for Reducing the Potential Risk of Mental Nerve Injury During Transoral Thyroidectomy[]]. World Journal of Surgery, 2020, 44(2): 371-377.

[7]. Zhang D, Fu Y, Dionigi G, et al. Human cadaveric model for studying the preservation of mental nerve during transoral endoscopic thyroidectomy[]]. Surgical and Radiologic Anatomy, 2020, 42(1): 55-62.

[8]. Camenzuli C, Wismayer P S, Agius J C, et al. Transoral Endoscopic Thyroidectomy: A Systematic Review of the Practice So Far.[]]. Jsls-journal of The Society of Laparoendoscopic Surgeons, 2018, 22(3).

[9]. Anuwong A, Sasanakietkul T, Jitpratoom P, et al. Transoral endoscopic thyroidectomy vestibular approach (TOETVA): indications, techniques and results[]]. Surgical Endoscopy and Other Interventional Techniques, 2018, 32(1): 456-465.

[10]. Zhang D, Park D, Sun H, et al. Indications, benefits and risks of transoral thyroidectomy[j]. Best Practice \& Research Clinical Endocrinology \& Metabolism, 2019, 33(4). [11]. Bellantone R D, Boscherini M, Lombardi C P, et al. Is the identification of the external branch of the superior laryngeal nerve mandatory in thyroid operation? Results of a prospective randomized study []]. Surgery, 2001, 130(6): 1055-1059.

[12]. ZHANG D, WANG T, KIM H, et al. Strategies for superior thyroid pole dissection in transoral thyroidectomy: a video operative guide []]. Surgical Endoscopy, 2020, 34(8): 3711-3721.

[13]. Delgadovargas B, Romerosalazar A L, Cobeta I, et al. Vocal Changes Following Thyroid Surgery: Prospective Study of Objective and Subjective Parameters[]]. Journal of Voice, 2017, 33(1): 27-32.

[14]. Barczynski M, Randolph G W, Cernea C R, et al. External branch of the superior laryngeal nerve monitoring during thyroid and parathyroid surgery: International Neural Monitoring Study Group standards guideline statement[]]. Laryngoscope, 2013.

[15]. Cheruiyot I, Kipkorir V, Henry B M, et al. Surgical anatomy of the external branch of the superior laryngeal nerve: a systematic review and meta-analysis[]]. Langenbeck's Archives of Surgery, 2018, 403(7): 811-823.

[16]. Ma Hongzhi, Fang Jugao, Hou Lizhen, et al. Study on the Physiological Anatomy and Function Protection of EBSLN during Thyroid Surgery []]. Int J Otolaryngol Head Neck Surg, 2020, 44(01):1-5. (in Chinese)

[17]. Alberto M, Lianos G D, Luigi B, et al. Intraoperative Neuromonitoring of the External Branch of the Superior Laryngeal Nerve during Thyroidectomy: The Need for Evidence-Based Data and Perioperative Technical/Technological Standardization[]]. 
ScientificWorldJournal, 2014, 2014:854-5.

[18]. Friedman M, Losavio P, Ibrahim $\mathrm{H}$, et al. Superior Laryngeal Nerve Identification and Preservation in Thyroidectomy[]]. Archives of Otolaryngology-head \& Neck Surgery, 2002, 128(3): 296-303.

[19]. Wang K, Cai H, Kong D, et al. The Identification, Preservation and Classification of the External Branch of the Superior Laryngeal Nerve in Thyroidectomy[]]. World Journal of Surgery, 2017, 41(10): 2521-2529.

[20]. Henry L R, Solomon N P, Howard R S, et al. The functional impact on voice of sternothyroid muscle division during thyroidectomy[]]. Annals of Surgical Oncology, 2008, 15(7): 2027-2033. 


\section{Figures}

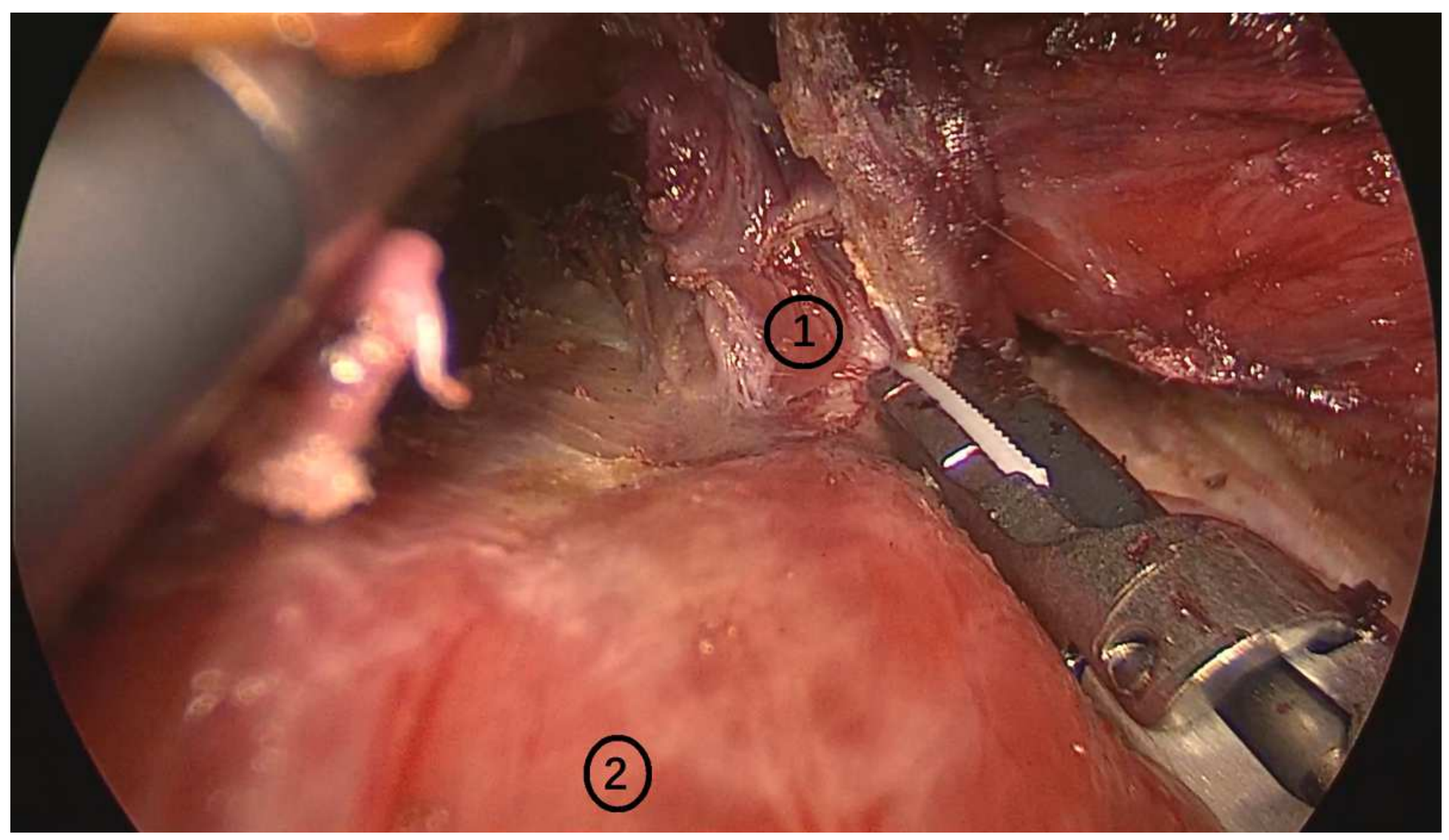

\section{Figure 1}

The sternothyroid muscle was transected at an attachment point on the thyroid cartilage, and was pushed to the sternal side, exposing the I obe of the thyroid gland. Here, $\nabla$ represents the transverse sternoth yroid muscle, and $\otimes$ represents the thyroid gland lobe. 


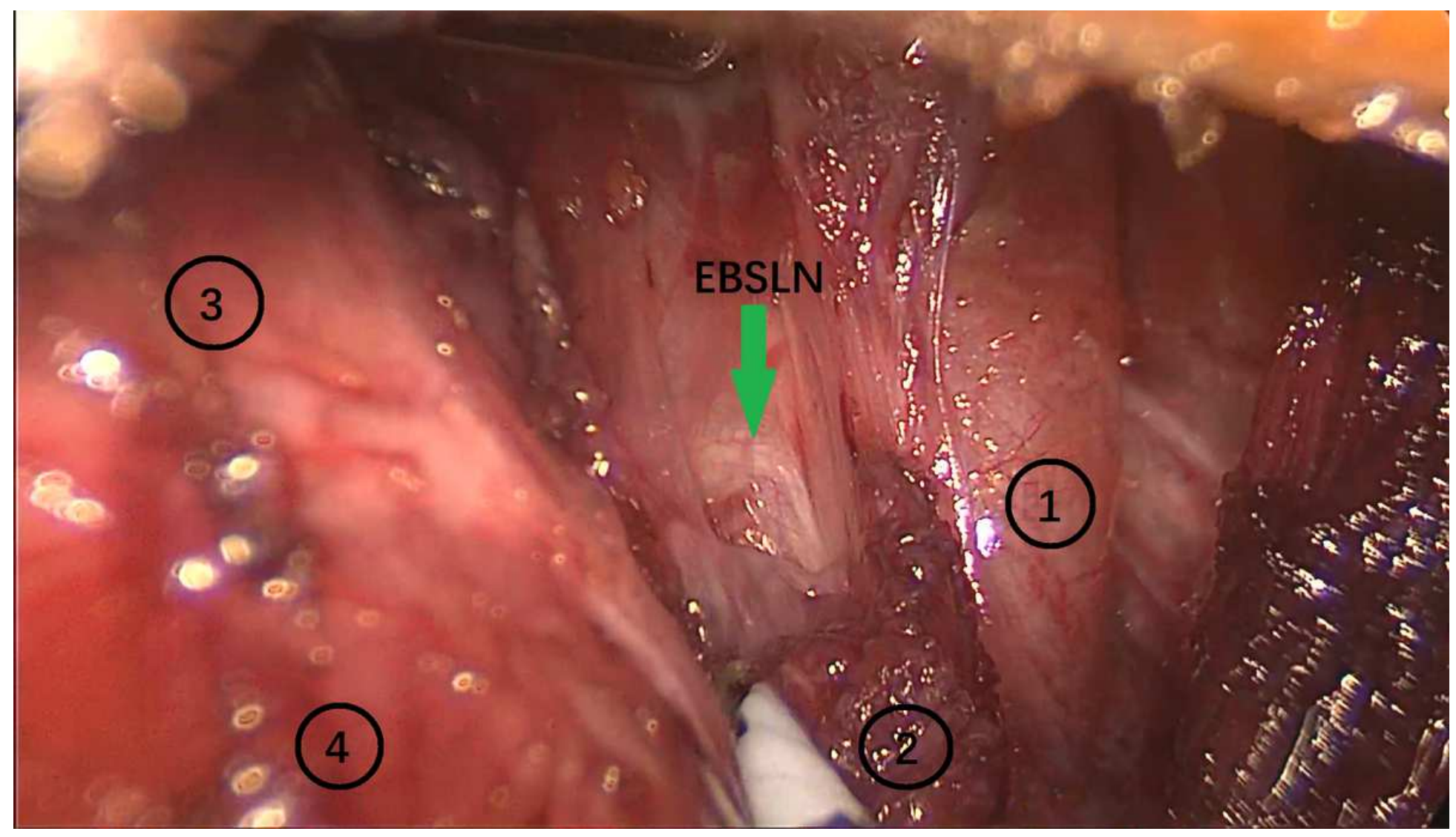

Figure 2

The thy roid gland was pulled outward and downward to expose Joll's space. In Joll's space, we looked

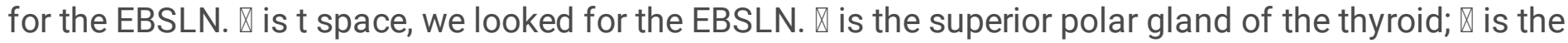
he superior polar gland of the thyroid; $\nabla$ is the transverse sternothtransverse sternothyroid muscle; $\nabla$ is the cricothyroid muscle (CTMyroid muscle; $\nabla$ is the cricothyroid muscle (CTM) and $\nabla$ is the throat.) and $\nabla$ is the throat. 

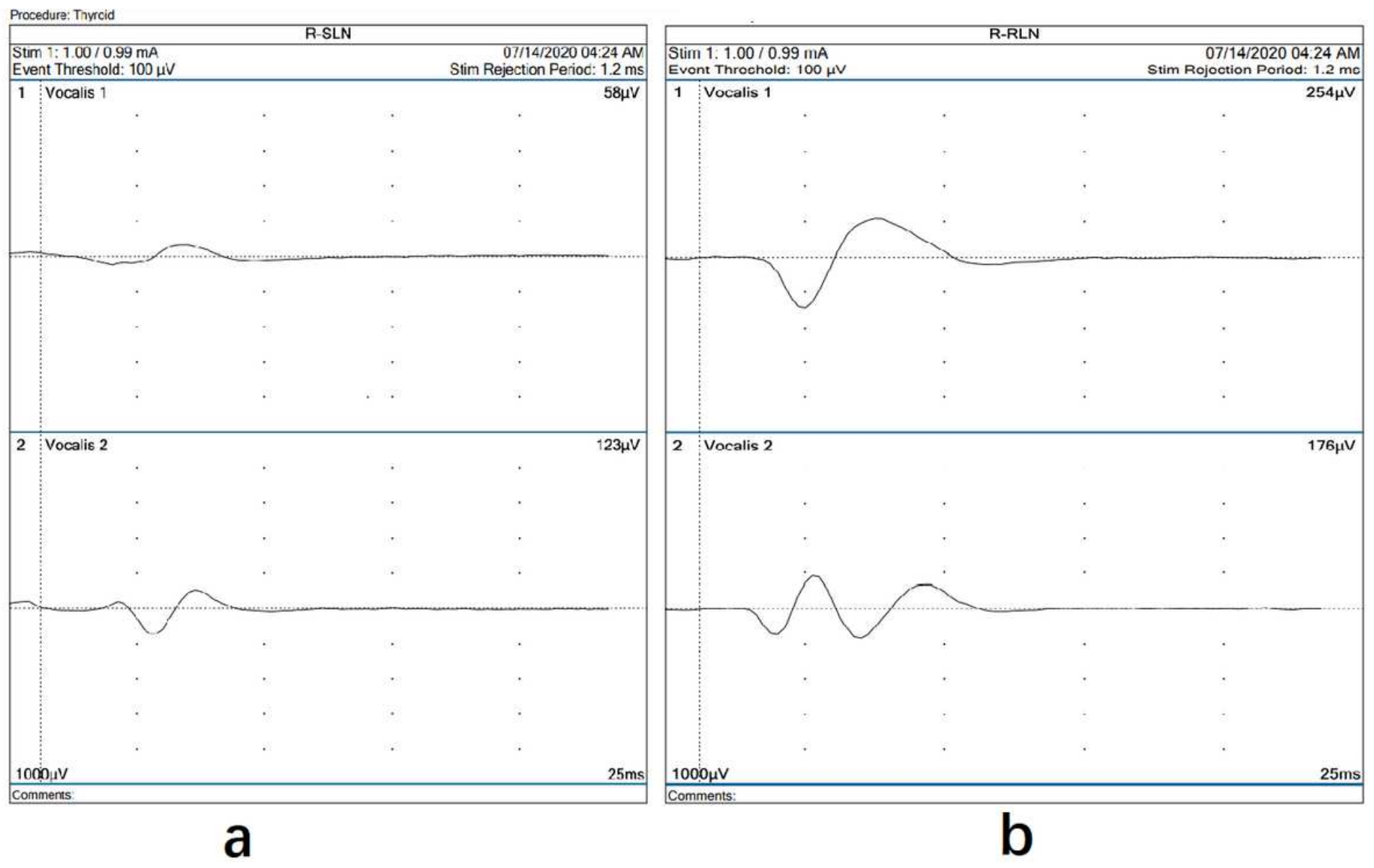

\section{Figure 3}

When IONM stimulated the EBSLN, CTM twitching was observed in all patients, and potentiometric w ave images were present in some patients (see Figure 3a), and the w aveform of th e EBLSN was completely different from that of the RLN (see Figure $3 b$ ). 

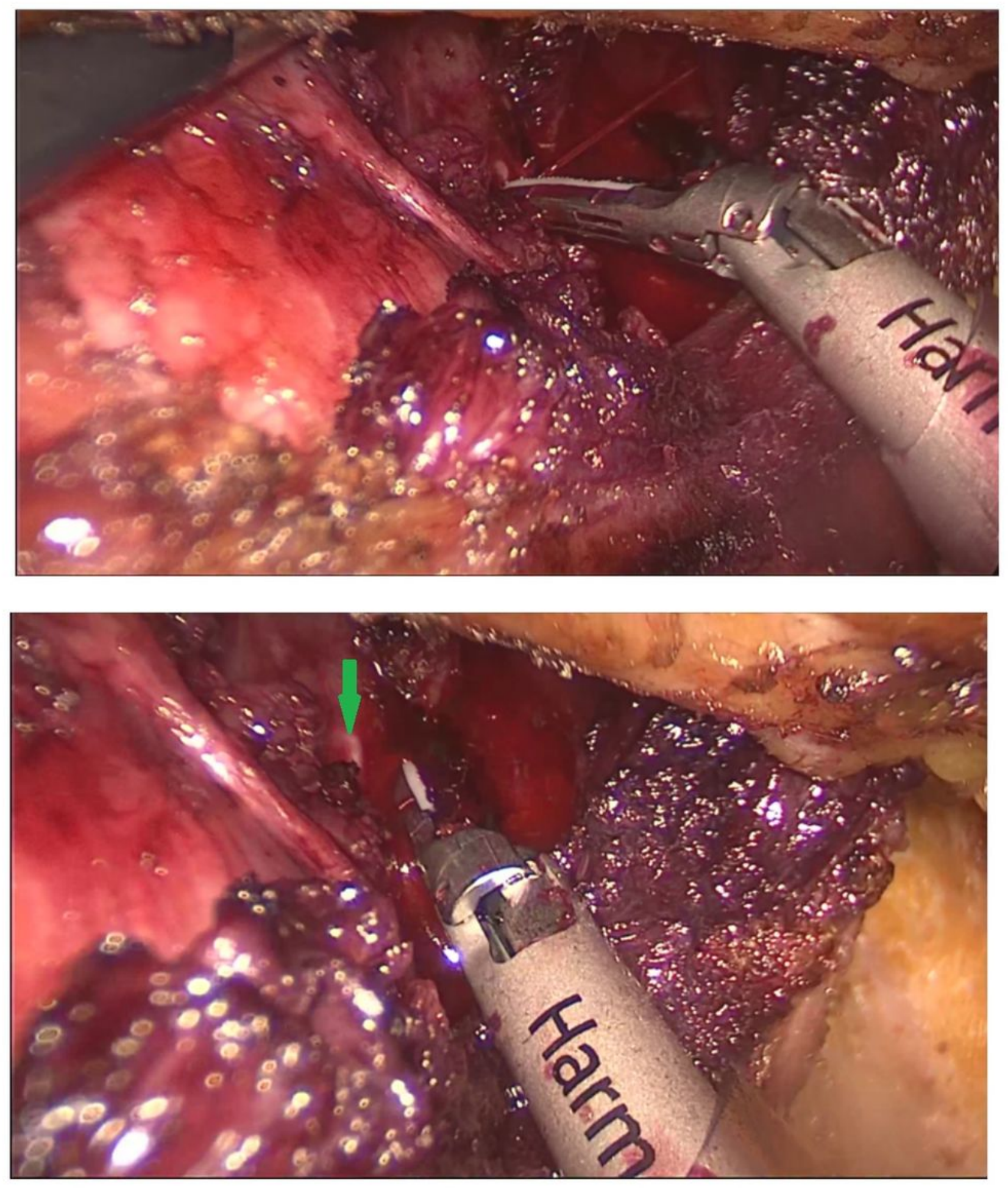

\section{Figure 4}

The bleeding point of the superior thyroid artery could be found under direct vision. The bleeding point could be clipped with an ultrasonic knif e, and the non fu nctional head of the ultrasonic knife could be kept away from the EBSLN to avoid injury to the EBSLN. The arrow in the figure indicates the EBSLN. 


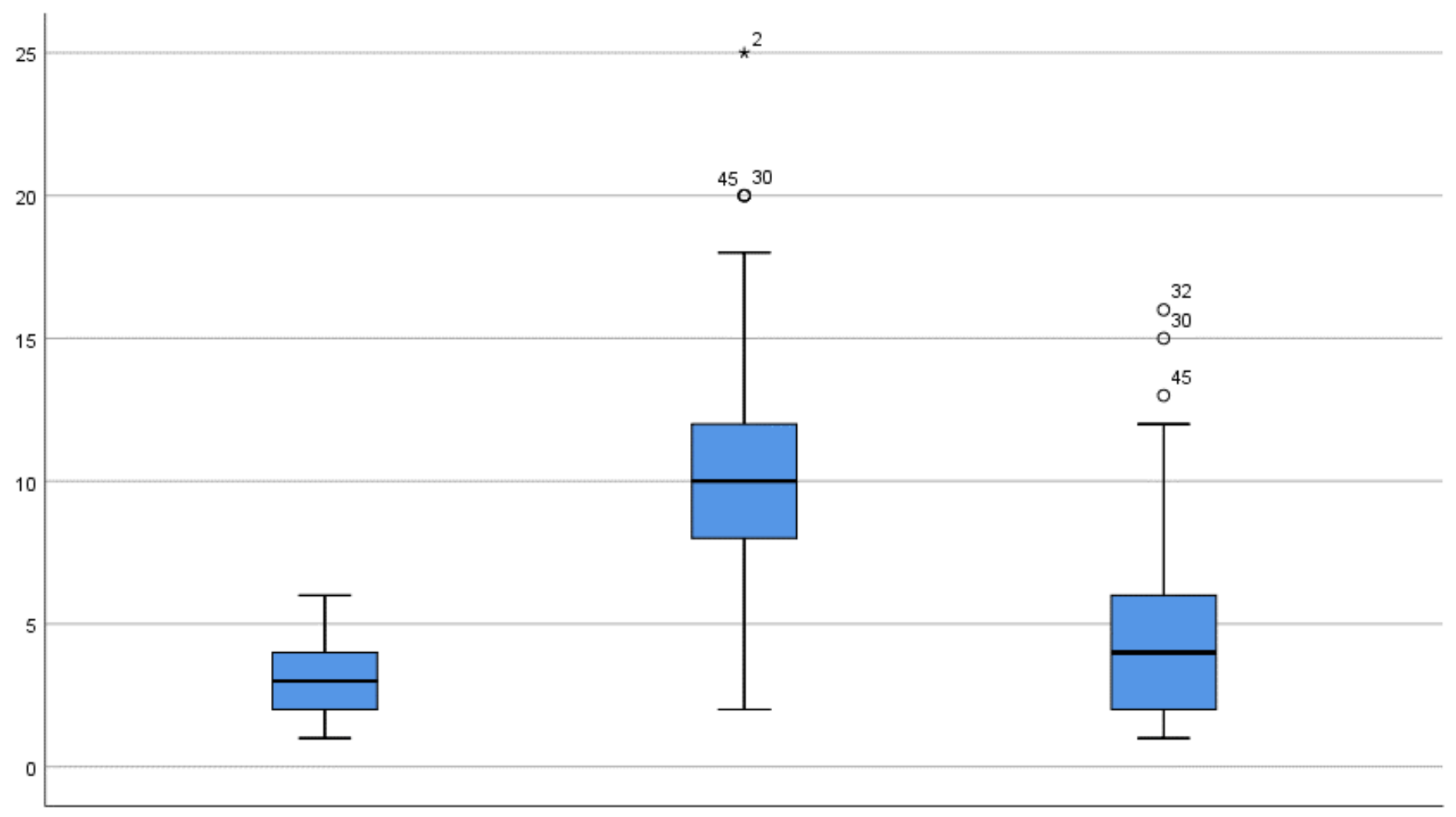

Preoperative One week after surgery Three weeks after surgery

Figure 5

Three weeks postoperatively, the vas t majority of patients returned to their preoperative status. 